\title{
Hamiltonian reduction of Einstein's equations without isometries
}

\author{
Jong Hyuk Yoon ${ }^{1, \star}$ \\ ${ }^{1}$ Department of Physics, Konkuk University, 120 Neungdong-ro, Gwangjin-gu, Seoul 05029, Korea
}

\begin{abstract}
I apply the Hamiltonian reduction procedure to 4-dimensional spacetimes without isometries and find privileged spacetime coordinates in which the physical Hamiltonian is expressed in terms of the conformal two metric and its conjugate momentum. Physical time is the area element of the cross section of null hypersurface, and the physical radial coordinate is defined by equipotential surfaces on a given spacelike hypersurface of constant physical time. The physical Hamiltonian is local and positive in the privileged coordinates. Einstein's equations in the privileged coordinates are presented as Hamilton's equations of motions obtained from the physical Hamiltonian.
\end{abstract}

\section{Introduction}

It is well-known that the true gravitational degrees of freedom of general relativity reside in the conformal two metric of the spatial cross section of null hypersurfaces[1, 2]. Eliminating unphysical degrees of freedom by identifying arbitrarily specifiable spacetime coordinates with certain functions in phase space and thereby presenting the theory in terms of physical degrees of freedom in the privileged coordinates, free from constraints, is known as the ADM Hamiltonian reduction[3-5]. Prof. Kuchař and others applied this procedure to spacetimes that admit two commuting Killing vector fields, known as midi-superspace[6-8], and showed that Einstein's theory was equivalent to cylindrical massless scalar field theory propagating in the $1+1$ dimensional Minkowski spacetime.

In this paper, we apply the ADM Hamiltonian reduction to general spacetimes of 4 dimensions under no symmetry assumption[9-13]. The area element of the spatial cross section of a null hypersurface emerges as the physical time, and the physical radial coordinate is defined by equipotential surfaces on a given spacelike hypersurface of constant physical time. We present the fully reduced physical Hamiltonian in these privileged coordinates[14], which turns out to be local and positive. The momentum constraints turn out to be simply the defining equations of the physical linear and angular momentum densities in term of the conformal two metric and its conjugate momentum[6]; hence, there remain no constraints to solve, and the theory becomes constraint-free. Moreover, we find that our Hamiltonian reduction is self-consistent because Hamilton's equations of motion obtained through this Hamiltonian reduction are identical to the Ricci-flat equations in the privileged coordinates. As a by-product of this Hamiltonian reduction, we found an independent proof of topological censorship[15-20], which followed directly from one of the Einstein's equations in these coordinates.

\section{The action in the $(2+2)$ formalism}

Let us recall that the metric in the $(2+2)$ decomposition[1, $2,9-13]$ of 4 dimensional spacetimes can be written as

$$
\begin{aligned}
d s^{2}= & 2 d u d v-2 h d u^{2}+\tau \rho_{a b}\left(d y^{a}+A_{+}^{a} d u+A_{-}^{a} d v\right) \\
& \times\left(d y^{b}+A_{+}^{b} d u+A_{-}^{b} d v\right) .
\end{aligned}
$$

The vector fields $\hat{\partial}_{ \pm}$defined as

$$
\hat{\partial}_{ \pm}:=\partial_{ \pm}-A_{ \pm}^{a} \partial_{a}
$$

where

$$
\partial_{+}=\partial / \partial u, \quad \partial_{-}=\partial / \partial v, \quad \partial_{a}=\partial / \partial y^{a} \quad(a=2,3),
$$

are horizontal vector fields orthogonal to the twodimensional spacelike surface $N_{2}$ generated by $\partial_{a}$. The inner products of the horizontal vector fields are given by

$$
\begin{aligned}
& <\hat{\partial}_{+}, \hat{\partial}_{+}>=-2 h, \quad<\hat{\partial}_{+}, \hat{\partial}_{-}>=1, \\
& <\hat{\partial}_{-}, \hat{\partial}_{-}>=0,
\end{aligned}
$$

which tells us that $\hat{\partial}_{-}$is a null vector field, and that $\hat{\partial}_{+}$has a norm $-2 h$, which can be either positive, negative, or zero, depending on the sign of $h$. In this paper, we choose the sign $-2 h>0$ so that $v=$ constant is a spacelike hypersurface. The metric on $N_{2}$ is $\tau \rho_{a b}$, where $\tau$ is the area element of $N_{2}$ and $\rho_{a b}$ is the conformal two metric with $\operatorname{det} \rho_{a b}=1$.

As was shown in Ref.[13], the Einstein's equations can be obtained from the variational principle of the following action integral:

$$
\begin{gathered}
S=\int d v d u d^{2} y\left\{\pi_{\tau} \dot{\tau}+\pi_{h} \dot{h}+\pi_{a} \dot{A}_{+}^{a}+\pi^{a b} \dot{\rho}_{a b}\right. \\
\left.-“ 1 " \cdot C_{-}-“ 0 " \cdot C_{+}-A_{-}^{a} C_{a}\right\},
\end{gathered}
$$


where the overdot $"=\partial_{-}$, and " $1 "$, " $0 "$ ", and $A_{-}^{a}$ are Lagrange multipliers that enforce the constraints $C_{-}=0$, $C_{+}=0$, and $C_{a}=0$, which are given by

$$
\begin{aligned}
& \text { (i) } C_{-}:=\frac{1}{2} \pi_{h} \pi_{\tau}-\frac{h}{4 \tau} \pi_{h}^{2}-\frac{1}{2 \tau} \pi_{h} D_{+} \tau+\frac{1}{2 \tau^{2}} \rho^{a b} \pi_{a} \pi_{b} \\
& -\frac{\tau}{8 h} \rho^{a b} \rho^{c d}\left(D_{+} \rho_{a c}\right)\left(D_{+} \rho_{b d}\right)-\frac{1}{2 h \tau} \rho_{a b} \rho_{c d} \pi^{a c} \pi^{b d} \\
& -\frac{1}{2 h} \pi^{a c} D_{+} \rho_{a c}-\tau \mathrm{R}_{(2)}+D_{+} \pi_{h}-\partial_{a}\left(\tau^{-1} \rho^{a b} \pi_{b}\right) \\
& \quad=0, \\
& \text { (ii) } C_{+}:=\pi_{\tau} D_{+} \tau+\pi_{h} D_{+} h+\pi^{a b} D_{+} \rho_{a b} \\
& -2 D_{+}\left(h \pi_{h}+D_{+} \tau\right)+2 \partial_{a}\left(h \tau^{-1} \rho^{a b} \pi_{b}+\rho^{a b} \partial_{b} h\right) \\
& =0,
\end{aligned}
$$

(iii) $C_{a}:=\pi_{\tau} \partial_{a} \tau+\pi_{h} \partial_{a} h+\pi^{b c} \partial_{a} \rho_{b c}-2 \partial_{b}\left(\rho_{a c} \pi^{b c}\right)$

$$
-D_{+} \pi_{a}-\partial_{a}\left(\tau \pi_{\tau}\right)=0
$$

respectively. Here, $\mathrm{R}_{(2)}$ is the scalar curvature of $N_{2}$, and the diff $N_{2}$-covariant derivative[13] of a tensor density $q_{a b}$ with weight $s$ is defined as

$$
\begin{gathered}
D_{+} q_{a b}:=\partial_{+} q_{a b}-\left[A_{+}, q\right]_{\mathrm{L} a b}=\partial_{+} q_{a b}-A_{+}^{c} \partial_{c} q_{a b} \\
\quad-q_{c b} \partial_{a} A_{+}^{c}-q_{a c} \partial_{b} A_{+}^{c}-s\left(\partial_{c} A_{+}^{c}\right) q_{a b},
\end{gathered}
$$

where $\left[A_{+}, q\right]_{\mathrm{L} a b}$ is the Lie derivative of $q_{a b}$ along $A_{+}:=$ $A_{+}{ }^{a} \partial_{a}$. For instance, the diff $N_{2}$-covariant derivatives of the area element $\tau$ and the conformal metric $\rho_{a b}$, which are a scalar density and a tensor density with weight 1 and -1 with respect to the diff $N_{2}$ transformations, respectively, are given by

$$
\begin{aligned}
D_{ \pm} \tau=\partial_{ \pm} \tau-A_{ \pm}^{a} \partial_{a} \tau-\left(\partial_{a} A_{ \pm}^{a}\right) \tau, \\
D_{ \pm} \rho_{a b}=\partial_{ \pm} \rho_{a b}-A_{ \pm}^{c} \partial_{c} \rho_{a b}-\rho_{c b} \partial_{a} A_{ \pm}^{c}-\rho_{a c} \partial_{b} A_{ \pm}^{c} \\
\\
+\left(\partial_{c} A_{ \pm}^{c}\right) \rho_{a b} .
\end{aligned}
$$

On the other hand, $h$ is a scalar under the $\operatorname{diff} N_{2}$ transformations, whose diff $N_{2}$-covariant derivative is given by

$$
D_{ \pm} h=\partial_{ \pm} h-A_{ \pm}^{a} \partial_{a} h,
$$

and the $\operatorname{diff} N_{2}$-covariant field strength $F_{+-}{ }^{a}$ is defined as

$$
\begin{aligned}
F_{+-}^{a}: & =\partial_{+} A_{-}^{a}-\partial_{-} A_{+}^{a}-\left[A_{+}, A_{-}\right]^{a} \\
& =\partial_{+} A_{-}^{a}-\partial_{-} A_{+}^{a}-A_{+}^{b} \partial_{b} A_{-}^{a}+A_{-}^{b} \partial_{b} A_{+}^{a} .
\end{aligned}
$$

The diff $N_{2}$-covariant derivatives of the conjugate momenta $\pi_{\tau}, \pi_{h}, \pi_{a}$, and $\pi^{a b}$, which are tensor densities of weights $0,1,1$ and 2 , respectively, are given by

$$
\begin{aligned}
D_{ \pm} \pi_{\tau}= & \partial_{ \pm} \pi_{\tau}-A_{ \pm}^{a} \partial_{a} \pi_{\tau}, \\
D_{ \pm} \pi_{h}= & \partial_{ \pm} \pi_{h}-A_{ \pm}^{c} \partial_{c} \pi_{h}-\left(\partial_{c} A_{ \pm}^{c}\right) \pi_{h}, \\
D_{ \pm} \pi_{a}= & \partial_{ \pm} \pi_{a}-A_{ \pm}^{c} \partial_{c} \pi_{a}-\pi_{c} \partial_{a} A_{ \pm}^{c}-\left(\partial_{c} A_{ \pm}^{c}\right) \pi_{a}, \\
D_{ \pm} \pi^{a b}= & \partial_{ \pm} \pi^{a b}-A_{ \pm}^{c} \partial_{c} \pi^{a b}+\pi^{c b} \partial_{c} A_{ \pm}^{a}+\pi^{a c} \partial_{c} A_{ \pm}^{b} \\
& -2\left(\partial_{c} A_{ \pm}^{c}\right) \pi^{a b} .
\end{aligned}
$$

We note that because $\rho_{a b}$ has a unit determinant, its derivatives satisfy the conditions

$$
\rho^{b c} \partial_{ \pm} \rho_{b c}=\rho^{b c} \partial_{a} \rho_{b c}=\rho^{b c} D_{ \pm} \rho_{b c}=0,
$$

and the conjugate momentum $\pi^{a b}$ is traceless,

$$
\rho_{a b} \pi^{a b}=0 .
$$

\section{Hamiltonian reduction I: $\tau$ as physical time}

Let us define a potential function $R$ and its conjugate momentum $\pi_{R}$ as

$$
\begin{aligned}
& \partial_{+} R:=-h \pi_{h}, \\
& \pi_{R}=-\partial_{+} \ln (-h),
\end{aligned}
$$

respectively. The transformation from $\left(h, \pi_{h}\right)$ to $\left(R, \pi_{R}\right)$ is a canonical transformation, as it changes the action integral by total derivatives only. If we impose the constraints $C_{+}=0$ and $C_{a}=0$ and choose the Lagrange multiplier $A_{-}^{a}=0$, then the action in Eq. (5) becomes

$$
\begin{aligned}
S=\int d v d u d^{2} y\left\{\pi_{\tau} \dot{\tau}+\pi_{R} \dot{R}+\pi_{a} \dot{A}_{+}^{a}+\pi^{a b} \dot{\rho}_{a b}-C_{-}\right\} \\
+ \text {total derivatives, }
\end{aligned}
$$

where the constraint $C_{-}$in the new variables is given by

$$
\begin{aligned}
C_{-} & =-\frac{1}{2 h} \pi_{\tau} \partial_{+} R-\frac{1}{4 h \tau}\left(\partial_{+} R\right)^{2}+\frac{1}{2 h \tau}\left(D_{+} \tau\right)\left(\partial_{+} R\right) \\
& -\frac{1}{h} D_{+}\left(\partial_{+} R\right)+\frac{1}{h}\left(\partial_{+} R\right) \partial_{+} \ln (-h) \\
& -\frac{1}{h}\left(\partial_{+} R\right) A_{+}^{a} \partial_{a} \ln (-h) \\
& -\tau R^{(2)}+\frac{1}{2 \tau^{2}} \rho^{a b} \pi_{a} \pi_{b}-\partial_{a}\left(\tau^{-1} \rho^{a b} \pi_{b}\right) \\
& -\frac{1}{2 h \tau} \rho_{a b} \rho_{c d} \pi^{a c} \pi^{b d}-\frac{\tau}{8 h} \rho^{a b} \rho^{c d}\left(D_{+} \rho_{a c}\right)\left(D_{+} \rho_{b d}\right) \\
& -\frac{1}{2 h} \pi^{a c} D_{+} \rho_{a c}=0 .
\end{aligned}
$$

The function $h$ still appears in Eq. (23), but as $h$ is related to $R$ and $\pi_{R}$ by Eqs. (20) and (21), the constraint function $C_{-}$given by Eq. (23) may be viewed as a function of the new variables $\left(\tau, Q^{I} ; \pi_{\tau}, \Pi_{I}\right)$, where $Q^{I}=\left(R, A_{+}^{a}, \rho_{a b}\right)$ and $\Pi_{I}=\left(\pi_{R}, \pi_{a}, \pi^{a b}\right)$. Notice that the first term in Eq. (23) is linear in $\pi_{\tau}$, and that all the remaining terms are independent of $\pi_{\tau}$. Thus, the equation of motion for $\tau$ is given by

$$
\dot{\tau}=\int d u d^{2} y \frac{\delta C_{-}}{\delta \pi_{\tau}}=-\frac{1}{2 h} \partial_{+} R .
$$

Now, recall that $\tau=\tau\left(v, u, y^{a}\right)$. If we solve this equation for $v$, then $v$ may be viewed as a function of $\left(\tau, u, y^{a}\right)$ and consequently, $\left(R, A_{+}^{a}, \rho_{a b}\right)$ may be regarded as functions of $\left(\tau, u, y^{a}\right)$. Therefore,

$$
\begin{aligned}
& \dot{R}=\dot{\tau} \partial_{\tau} R, \dot{A}_{+}^{a}=\dot{\tau} \partial_{\tau} A_{+}^{a}, \dot{\rho}_{a b}=\dot{\tau} \partial_{\tau} \rho_{a b}, \\
& C_{-}=-\left(\frac{2 h}{\partial_{+} R}\right) \dot{\tau} C_{-},
\end{aligned}
$$

because $\partial u / \partial v=\partial y^{a} / \partial v=0$. Then, the action in Eq. (22) becomes

$$
\begin{aligned}
& S=\int d v d u d^{2} y \dot{\tau}\left\{\pi_{\tau}+\pi_{R} \partial_{\tau} R+\pi_{a} \partial_{\tau} A_{+}^{a}\right. \\
& \left.+\pi^{a b} \partial_{\tau} \rho_{a b}+\left(\frac{2 h}{\partial_{+} R}\right) C_{-}\right\} \\
& =\int d \tau d u d^{2} y\left\{\pi_{R} \partial_{\tau} R+\pi_{a} \partial_{\tau} A_{+}^{a}+\pi^{a b} \partial_{\tau} \rho_{a b}-C_{(1)}\right\} \\
& =\int d \tau d u d^{2} y\left\{\sum_{I} \Pi_{I} \partial_{\tau} Q^{I}-C_{(1)}\right\}
\end{aligned}
$$




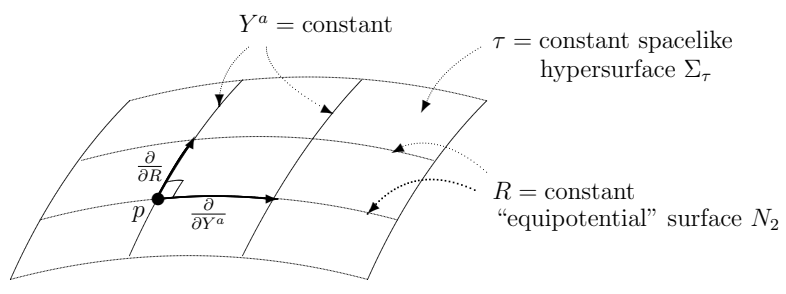

Figure 1. On $R=$ constant surface $N_{2}$ on $\Sigma_{\tau}, Y^{a}=$ constant is chosen to be normal to $N_{2}$ at each point $p$ on $\Sigma_{\tau}$. In this case the "shift" vector $A_{+}^{a}$ is zero at $p$.

where we replaced $d v \dot{\tau}$ by $d \tau$ in the second line, and $C_{(1)}$ is defined as

$$
C_{(1)}=-\left(\frac{2 h}{\partial_{+} R}\right) C_{-}-\pi_{\tau} .
$$

Notice that if we impose the constraint $C_{-}=0$, the function $C_{(1)}$ becomes

$$
C_{(1)}=-\pi_{\tau}
$$

\section{Hamiltonian reduction II: Fixation of $u=R$ and $y^{a}=Y^{a}$ such that $A_{+}^{a}=0$}

The second step in the Hamiltonian reduction consists of identifying arbitrarily specifiable coordinates $u$ and $y^{a}$ as

$$
\begin{aligned}
& u=R, \\
& y^{a}=Y^{a}
\end{aligned}
$$

such that the "shift" vector $A_{+}^{a}$ is zero, i.e.,

$$
A_{+}^{a}=0 \text {. }
$$

For the class of spacetimes whose spatial topology is either $N_{2} \times \mathbb{R}$ or $N_{2} \times S^{1}$, where $N_{2}$ is a compact two-dimensional space with the genus $g$, the condition in Eq. (31) can always be met by relabeling $N_{2}$ such that $Y^{a}=$ constant is normal to $u=$ constant at each point on $\Sigma_{\tau}$. If we continue to label $N_{2}$ this way, then the two dimensional "shift" $A_{+}^{a}$ can always be made zero for the class of spacetimes under consideration (see Fig. 1). Thus, we will work in the coordinates $X^{A}:=\left(\tau, R, Y^{a}\right)$, which satisfy the coordinate conditions

$$
\frac{\partial X^{A}}{\partial X^{B}}=\delta_{B}^{A} .
$$

Then, it follows from Eq. (24) that

$$
\dot{\tau}=-\frac{1}{2 h}>0,
$$

which means that $\tau$ increases monotonically along the outgoing null vector field.

Hamilton's equations of motion follow from the variational principle of the action integral in Eq. (26):

$$
\begin{aligned}
& \partial_{\tau} Q^{I}=\left.\int_{\Sigma_{\tau}} d u d^{2} y \frac{\delta C_{(1)}}{\delta \Pi_{I}}\right|_{u=R, y^{a}=Y^{a},} \\
& \partial_{\tau} \Pi_{I}=-\left.\int_{\Sigma_{\tau}} d u d^{2} y \frac{\delta C_{(1)}}{\delta Q^{I}}\right|_{u=R, y^{a}=Y^{a}},
\end{aligned}
$$

where $Q^{I}=\left(R, A_{+}^{a}, \rho_{a b}\right), \Pi_{I}=\left(\pi_{R}, \pi_{a}, \pi^{a b}\right)$, and $\Sigma_{\tau}$ is a spacelike hypersurface defined by $\tau=$ constant.

\section{Main results}

In the following, we present the main results of this paper. Einstein's evolution equations I

1. $\frac{\partial R}{\partial \tau}=0=$

$$
\tau \mathrm{R}_{(2)}=\frac{1}{2} \tau^{-2} \rho^{a b} \pi_{a} \pi_{b}-\partial_{a}\left(\tau^{-1} \rho^{a b} \pi_{b}\right)
$$

(topological censorship)

2. $\frac{\partial A_{+}^{a}}{\partial \tau}=0 \Rightarrow$ $\tau^{-1} \pi_{a}=-\partial_{a} \ln (-h)$ (superpotential)

3. $\frac{\partial \tau}{\partial \tau}=1$ (trivial)

4. $\frac{\partial \ln (-h)}{\partial \tau}=H-\frac{1}{\tau}$ (superpotential)

5. $\frac{\partial \pi_{a}}{\partial \tau}=2 \tau^{-1} \pi_{a}+\left(\pi^{b c}+\frac{\tau}{2} \rho^{b d} \rho^{c e} \partial_{R} \rho_{d e}\right) \partial_{a} \rho_{b c}$

$-\partial_{b}\left(2 \pi^{b c} \rho_{a c}+\tau \rho^{b c} \partial_{R} \rho_{a c}\right)$

(evolution equation of $\pi_{a}$ )

6. $\frac{\partial \pi_{\tau}}{\partial \tau}=\frac{1}{2} \tau^{-2}+\tau^{-2} \rho_{a b} \rho_{c d} \pi^{a c} \pi^{b d}$

$-\frac{1}{4} \rho^{a b} \rho^{c d}\left(\partial_{R} \rho_{a c}\right)\left(\partial_{R} \rho_{b d}\right)-2 \tau^{-2} \partial_{a}\left(h \rho^{a b} \pi_{b}\right)$

(evolution equation of $\pi_{\tau}$ )

Einstein's constraint equations

7. $C_{-}=0 \Rightarrow \pi_{\tau}=-H+2 \partial_{R} \ln (-h)$

$-2 h\left\{\tau \mathrm{R}^{(2)}-\frac{1}{2 \tau^{2}} \rho^{a b} \pi_{a} \pi_{b}+\frac{\partial}{\partial Y^{a}}\left(\tau^{-1} \rho^{a b} \pi_{b}\right)\right\}$

(definition of physical Hamiltonian)

8. $C_{+}=0 \Rightarrow \pi_{R}=-\pi^{a b} \partial_{R} \rho_{a b}$

(definition of physical linear momentum)

9. $C_{a}=0 \Rightarrow \tau^{-1} \pi_{a}=-\pi^{b c} \partial_{a} \rho_{b c}+2 \partial_{b}\left(\pi^{b c} \rho_{a c}\right)$ $-\tau \partial_{a}\left(H+\pi_{R}\right)$

(definition of physical angular momentum)

Superpotential $\ln (-h)$

$$
\begin{aligned}
& \text { 10. } \partial_{\tau} \ln (-h)=H-\tau^{-1} \\
& \text { 11. }-\partial_{R} \ln (-h)=\pi_{R} \\
& \text { 12. }-\partial_{a} \ln (-h)=\tau^{-1} \pi_{a}
\end{aligned}
$$

Integrability conditions

$$
\begin{aligned}
& \text { 13. } \partial_{R}\left(\tau^{-1} \pi_{a}\right)=\partial_{a} \pi_{R} \\
& \text { 14. } \partial_{\tau} \pi_{R}=-\partial_{R} H \\
& \text { 15. } \partial_{\tau}\left(\tau^{-1} \pi_{a}\right)=-\partial_{a} H .
\end{aligned}
$$

In the above equations, $H$ is defined by

$$
\begin{aligned}
H= & \frac{1}{\tau} \rho_{a b} \rho_{c d} \pi^{a c} \pi^{b d}+\frac{1}{4} \tau \rho^{a b} \rho^{c d}\left(\partial_{R} \rho_{a c}\right)\left(\partial_{R} \rho_{b d}\right) \\
& +\pi^{a c} \partial_{R} \rho_{a c}+\frac{1}{2 \tau} \geq \frac{1}{2 \tau} .
\end{aligned}
$$

The evolution equations of $\rho_{a b}$ and $\pi^{a b}$ can be found from the reduced action principle

$$
S^{*}=\int_{\Sigma_{\tau}} d R d^{2} Y\left\{\pi^{a b} \partial_{\tau} \rho_{a b}-C_{(1)}^{*}\right\},
$$


where $C_{(1)}^{*}$ is the restriction of $C_{(1)}$ to the coordinates $u=R$ and $y^{a}=Y^{a}$ :

$$
\begin{aligned}
& C_{(1)}^{*}:=\left.C_{(1)}\right|_{u=R, y^{a}=Y^{a}}=-\pi_{\tau} \\
& =H-2 \partial_{R} \ln (-h) .
\end{aligned}
$$

Einstein's evolution equations II

$$
\begin{aligned}
\text { 16. } \frac{\partial \rho_{a b}}{\partial \tau}=\int_{\Sigma_{\tau}} d R d^{2} Y \frac{\delta C_{(1)}^{*}}{\delta \pi^{a b}} \\
\text { 17. } \frac{\partial \pi^{a b}}{\partial \tau}=-\int_{\Sigma_{\tau}} d R d^{2} Y \frac{\delta C_{(1)}^{*}}{\delta \rho_{a b}} .
\end{aligned}
$$

The spacetime metric in these privileged coordinates becomes

$$
d s^{2}=-4 h d R d \tau-2 h d R^{2}+\tau \rho_{a b} d Y^{a} d Y^{b} .
$$

\section{6 discussion}

Let us discuss some key features of the Hamiltonian reduction discussed in this article.

(i) First of all, let us mention that the whole set of equations summarized in Section 5 is identical to the vacuum Einstein's equations

$$
\mathrm{R}_{A B}=0
$$

for spacetimes whose metric is given by Eq. (56). Thus, the whole procedure of the Hamiltonian reduction respects general covariance, as it must, even though the final theory is written in the privileged coordinates.

(ii) The integral of Eq. (36) over a closed two surface $N_{2}$ becomes

$$
\int_{N_{2}} d^{2} Y \tau^{-2} \rho^{a b} \pi_{a} \pi_{b}=16 \pi(1-g) \geq 0,
$$

where $g$ is the genus of $N_{2}$. This identity states that, as long as the out-going null hypersurface forms a congruence of null geodesics which admits a cross section, the spatial topology of that null hypersurface is either a two sphere or a torus. This is a remarkably simple proof of topological censorship, as it does not rely on assumptions such as global hyperbolicity, asymptotic conditions, energy conditions, and so on, either inside or outside the null hypersurface, which are normally assumed in the literature[15-20], although a condition that no caustics exist on the null hypersurface is necessary for the existence of well-defined cross sections of the null congruences.

(iii) The spatial integral of $C_{(1)}^{*}$ defined in Eq. (53) is the sought-for physical Hamiltonian $K$ of vacuum spacetimes, haha

$$
K:=\int_{\Sigma_{\tau}} d R d^{2} Y H \geq 0
$$

which is positive-definite in the privileged coordinates.

(iv) The logarithm of the conformal factor in the $(\tau, R)$ subspace is the superpotential $\ln (-h)$, whose gradients yield $\left(H-\tau^{-1},-\pi_{R},-\tau^{-1} \pi_{a}\right)$ through Eqs. (45), (46), and
(47), respectively. The superpotential[6] is a local function of $X^{A}=\left(\tau, R, Y^{a}\right)$, and is determined by the line integral

$$
\left.\ln \frac{h(X)}{h\left(X_{0}\right)}=\int_{X_{0}}^{X}\left(H-\tau^{-1}\right) d \tau-\pi_{R} d R-\tau^{-1} \pi_{a} d Y^{a}\right\}
$$

along any contour from $X_{0}$ to $X$ in a given spacetime.

(v) The integrability conditions in Eqs. (48), (49), and (50) are the consistency conditions, which follow from the definition of the superpotential $\ln (-h)$.

(vi) We would like to emphasize that all the constraint equations, $C_{ \pm}=0$ and $C_{a}=0$ in the privileged coordinates, are solved in such a way that the constraints simply turn out to be the defining equations of the physical Hamiltonian density $\pi_{\tau}$, the linear momentum density $\pi_{R}$, and the angular momentum density $\tau^{-1} \pi_{a}$, as is summarized in Eqs. (42), (43), and (44), respectively. Namely, $\pi_{R}$ and $H$ given by Eqs. (43) and (51) are functions of $\left(\tau ; \rho_{a b}, \pi^{a b}\right)$, and the superpotential $\ln (-h)$ given by Eq. (60) is a function of $H, \pi_{R}$, and $\tau^{-1} \pi_{a}$, which is again determined by $\left(\tau ; \rho_{a b}, \pi^{a b}\right)$, up to an overall constant factor. Thus, all the terms on the right-hand sides of Eqs. (42), (43), and (44) are functions of $\left(\tau ; \rho_{a b}, \pi^{a b}\right)$. This means that the constraints are completely solved for $\pi_{\tau}, \pi_{R}$, and $\tau^{-1} \pi_{a}$ in terms of the free, unconstrained data $\left(\rho_{a b}, \pi^{a b}\right)$.

(vii) If we define the total linear momentum $\Pi_{R}$ and total angular momentum $\Pi_{a}$ as

$$
\Pi_{R}:=\int_{\Sigma_{\tau}} d R d^{2} Y \pi_{R}, \quad \Pi_{a}:=\int_{\Sigma_{\tau}} d R d^{2} Y \tau^{-1} \pi_{a},
$$

then we find, by integrating Eqs. (43) and (44),

$$
\begin{aligned}
& \Pi_{R}=-\int_{\Sigma_{\tau}} d R d^{2} Y \pi^{b c} \partial_{R} \rho_{b c}, \\
& \Pi_{a}=-\int_{\Sigma_{\tau}} d R d^{2} Y \pi^{b c} \frac{\partial}{\partial Y^{a}} \rho_{b c} .
\end{aligned}
$$

Eqs. (62) and (63) show that $\Pi_{R}$ and $\Pi_{a}$ are the generating functions of translations of $\rho_{a b}$ and $\pi^{a b}$ along $\partial_{R}$ and $\partial / \partial Y^{a}$. This justifies our interpretations of $\Pi_{R}$ and $\Pi_{a}$ as the total linear and angular momentum carried by the conformal two metric, respectively. Moreover, if no spatial boundaries exist, then by virtue of the integrability conditions in Eqs. (49) and (50), $\Pi_{R}$ and $\Pi_{a}$ are conserved in the time $\tau$.

\section{References}

[1] R. Sachs, Proc. Roy. Soc. A 270, 103 (1962).

[2] R. A. d'Inverno and J. Stachel, J. Math. Phys. 19 (12), 2447 (1978).

[3] R. Arnowitt, S. Deser, and C. W. Misner, Phys. Rev. 117, 1595 (1960).

[4] C. Rovelli and L. Smolin, Phys. Rev. Lett. 72, 446 (1994).

[5] J. D. Brown and K. V. Kuchař, Phys. Rev. D 51, 5600 (1995).

[6] K. V. Kuchař, Phys. Rev. D 4, 955 (1971).

[7] V. Husain, Phys. Rev. D 50, 6207 (1994). 
[8] J. D. Romano and C. G. Torre, Phys. Rev. D 53, 5634 (1996).

[9] Y. M. Cho, Q. H. Park, K. S. Soh and J. H. Yoon, Phys. Lett. B 286, 251 (1992).

[10] J. H. Yoon, Phys. Lett. B 308, 240 (1993).

[11] J. H. Yoon, Phys. Lett. B 451, 296 (1999).

[12] J. H. Yoon, J. Korean Phys. Soc. 34, 108 (1999).

[13] J. H. Yoon, Phys. Rev. D 70, 084037 (2004).

[14] V. Husain and T. Pawłowski, Phys. Rev. Lett. 108, 141301 (2012).
[15] S. W. Hawking, Comm. Math. Phys. 25, 152 (1972).

[16] J. L. Friedman, K. Schleich, and D. M. Witt, Phys. Rev. Lett. 71, 1486 (1993).

[17] P. T. Chruściel and R. M. Wald, Class. Quantum Grav. 11, L147 (1994).

[18] T. Jacobson, and S. Venkataramani, Class. Quant. Grav. 12, 1055 (1995).

[19] G. J. Galloway, Class. Quant. Grav. 13, 1471 (1996).

[20] G. J. Galloway, K. Schleich, D. M. Witt, and E. Woolgar, Phys. Rev. D 60, 104039 (1999). 